Sureda-Negre, J., Cerdà-Navarro, A., Calvo-Sastre, A., y Comas-Forgas, R. (2020). Las conductas fraudulentas del alumnado universitario español en las evaluaciones: valoración de su gravedad y propuestas de sanciones a partir de un panel de expertos. Revista de Investigación Educativa, 38(1), 201-219.

DOI: http://dx.doi.org/10.6018/rie.358781

\title{
Las conductas fraudulentas del alumnado universitario español en las evaluaciones: valoración de su gravedad y propuestas de sanciones a partir de un panel de expertos
}

\author{
Misconduct of Spanish university students in evaluations:An \\ assessment of its severity and proposals for sanctions from an \\ experts' panel
}

\author{
Jaume Sureda-Negre,Antoni Cerdà-Navarro,Aina Calvo-Sastre y Rubén Comas-Forgas \\ Universitat de les Illes Balears
}

\begin{abstract}
Resumen
Este trabajo se centra en el fraude en las evaluaciones por parte del alumnado universitario español y su reglamentación por parte de las universidades como dispositivo para combatirlo. A partir del análisis de la literatura y la consulta a un panel de expertos mediante la administración de un cuestionario en dos rondas se identifican los principales comportamientos fraudulentos en las evaluaciones. Posteriormente, a partir de las opiniones de los expertos se determina, por una parte, el nivel de gravedad de cada uno de los comportamientos fraudulentos y, por otra, el tipo de sanciones aplicables según su gravedad. El análisis del grado de concordancia de los expertos en torno a estas cuestiones sugiere que, si bien hay algunos elementos que presentan un consenso significativo, continúan habiendo importantes divergencias en algunos criterios fundamentales a la hora de valorar y sancionar los comportamientos fraudulentos del alumnado en las evaluaciones. Los resultados ofrecidos pueden ser de utilidad para la elaboración y desarrollo de políticas educativas contra la deshonestidad académica basadas en dispositivos normativos.

Palabras clave: enseñanza superior; engaño; política educativa; plagio.
\end{abstract}

Correspondencia: Rubén Comas-Forgas rubencomas@uib.es Campus UIB Edificio A. Turmeda 185, Palma CP:07122. 


\begin{abstract}
This paper focuses on fraud detection in assessment tests taken by Spanish university students and its consequent regulation in university contexts as a device to combat it. Building on an analysis of the existing literature and a consultation of a panel of experts through a questionnaire in two rounds, the main areas of fraudulent behaviour are identified. Based on the experts' opinion, the severity of each fraudulent act and the subsequent sanction to be applied are determined thereafter. The degree of congruence on the experts' response suggests that, despite significant consensus on some elements, there continue to be important divergences in some fundamental criteria when assessing and punishing the fraudulent behaviour of students in evaluations. The results offered can be useful for the development and implementation of educational policies against academic dishonesty based on regulatory devices.

Keywords: higher education; cheating; educational policy; plagiarism.
\end{abstract}

\title{
Introducción
}

Aunque la rectitud, probidad, honestidad y equidad (entre otros valores) constituyen principios básicos de la vida universitaria, son numerosas las evidencias que ponen de manifiesto cómo las prácticas corruptas están muy presentes en las instituciones de enseñanza superior (Chapman \& Lindner, 2016; Denisova-Schmidt, 2017; Hallak \& Poisson, 2007; Heyneman, Kathryn, Anderson \& Muraliyeva, 2008; Mohamedbhai, 2016; Osipian 2007; Sweeney, Despota \& Lindner, 2013).

Una de las manifestaciones más extendidas de la deshonestidad académica por parte del alumnado universitario consiste en las diversas formas de engaño en los procesos de evaluación: exámenes, trabajos, tesis, etc. El fraude académico en los procesos de evaluación -una de las principales formas de corrupción junto a la malversación, el soborno, la extorsión y el favoritismo- puede definirse como "any prescribed action taken in connection with an examination or test that attempts to gain unfair advantage" (Hallak \& Poisson, 2007, p.231). La prevalencia de este fenómeno ha sido ampliamente documentada en muchos países (Curtis \& Clare, 2017; Ercegovac \& Richardson, 2004; ETINED, 2018; Haswell, Jubb \& Wearing, 1999; Hu \& Lei, 2015; Ma, McCabe \& Liu, 2013), también en España (Comas \& Sureda, 2016; Comas, Sureda, Casero \& Morey, 2011; Foltynek, 2013).

Combatir la deshonestidad en las evaluaciones constituye un reto para las instituciones de enseñanza superior. Para ello han puesto en marcha dispositivos que, partiendo de la premisa de un necesario enfoque holístico (Macdonald \& Carroll, 2006), se pueden clasificar en tres categorías: los formativos; los de detección y, finalmente, los normativos o reguladores (Comas, 2009).

Los dispositivos formativos se concretan en servicios, actividades y recursos (cursos, seminarios, tutoriales, folletos, "writing centres", etc.) orientados a que el alumnado adquiera las competencias y los conocimientos que contribuyan a no incurrir en este tipo de prácticas deshonestas (Belter \& du Pré, 2009; Estow, Lawrence \& Adams, 2011). Por otra parte, los dispositivos de detección son herramientas tecnológicas utilizadas para detectar, principalmente, los casos de plagio (Kumar \& Tripathi, 2017; Ledwith \& Risquez, 2008; Youmans, 2011). Finalmente, los mecanismos normativos 
consisten en disposiciones reguladoras en las que se señalan los tipos de conductas deshonestas, así como las sanciones aplicables si se incurre en ellas (Sureda, Reynés \& Comas, 2016).

Este trabajo se focaliza en los dispositivos normativos de las universidades españolas. Cabe remarcar que España, donde la prevalencia de la deshonestidad es alta (Comas et al., 2011; Comas, Sureda \& Salva, 2010), es uno de los países europeos en los que el alumnado universitario recibe menos formación sobre la práctica deshonesta más extendida: el plagio (Foltynek, 2013); un país en el que las normativas universitarias sobre el tema son escasas y poco precisas (Sureda et al., 2016), y en el que la presencia de estas cuestiones en documentos como las guías docentes es exigua (Cebrian-Robles, Raposo-Rivas \& Sarmiento-Campos, 2016).

\section{Reglamentación contra el fraude académico en España}

Las normativas a favor de la honestidad en las evaluaciones han sido escasamente desarrolladas en España (Sureda et al., 2016), pudiéndose clasificar en dos categorías: las generales y las específicas de cada centro. Entre las generales destaca el Real Decreto 1791/2010 por el que se aprobó el "Estatuto del Estudiante Universitario" (2010, diciembre 30) y que regula las obligaciones y derechos de los estudiantes. En el artículo 13 se especifican los deberes del alumnado, señalándose que los estudiantes deberán "Abstenerse de la utilización o cooperación en procedimientos fraudulentos en las pruebas de evaluación, en los trabajos que se realicen o en documentos oficiales de la universidad". Por otra parte -y como única referencia a posibles medidas para evitar el fraude en las evaluaciones- en el artículo 25 se establece que "en cualquier momento de las pruebas de evaluación, el profesor podrá requerir la identificación de los estudiantes asistentes". En otro artículo, el 63, se reseña que "la honradez, la veracidad, el rigor, la justicia, la eficiencia, el respeto y la responsabilidad" deberán presidir las actuaciones de los universitarios.

Conviene saber que, hasta el momento, no existe una norma general de régimen disciplinario para el alumnado universitario español promulgada con posterioridad a la Constitución de 1978, con lo que sigue vigente el Reglamento de disciplina académica (1954, octubre 12) aprobado por decreto de 8 de septiembre de 1954. En todo caso la aplicación de este Reglamento tiene que hacerse a la luz de la Constitución y han de entenderse derogados tácitamente aquellos aspectos que la contradicen.

En este contexto, y al amparo de las previsiones de la Ley Orgánica 6/2001 (2001, diciembre, 21), concretamente del artículo 2 en el que se reconoce a las universidades la facultad de elaborar normas de régimen interno, y del artículo 46 que señala que "Los Estatutos y normas de organización y funcionamiento desarrollarán los derechos y los deberes de los estudiantes, así como los mecanismos para su garantía", al amparo de estas previsiones las Universidades españolas van reglamentado el tema del fraude con diversos tipos de normas para su alumnado.

El análisis de las normas propias de las universidades españolas (Sureda et al., 2016) han puesto de manifiesto un tratamiento superficial de la deshonestidad y una gran disparidad a la hora de establecer sanciones. Este contexto pone de manifiesto la pertinencia de obtener conocimientos que faciliten que las universidades españo- 
las puedan elaborar y aplicar unos reglamentos que, juntamente con dispositivos formativos y de detección, contribuyan a la promoción de la integridad académica en el contexto universitario.

\section{Objetivos}

La finalidad de este trabajo consiste en obtener conocimientos que faciliten la puesta en marcha de dispositivos normativos para luchar contra el fraude en las evaluaciones por parte del alumnado universitario español. La consecución de esta finalidad se aborda contestando a tres cuestiones:

1) ¿Cuáles son las principales conductas fraudulentas en las evaluaciones del alumnado?

2) ¿Cuál es el nivel de gravedad de cada uno de estos comportamientos fraudulentos?

3) ¿Qué sanciones habría que aplicar a las faltas según su nivel de gravedad?

\section{Método}

Para dar respuesta a las cuestiones planteadas se optó por la consulta mediante cuestionario on-line a un panel de expertos formado por miembros de tres colectivos: presidentes o secretarios de los consejos sociales de las universidades españolas; miembros de la Asociación Española de Derecho Universitario (AEDUN) y profesores universitarios con experiencia de gestión académica.

El panel quedó constituido por 62 personas cuyas características de señalan en los cuadros 1,2 y 3 .

Cuadro 1

Categoría profesional de los participantes.

\begin{tabular}{lcc}
\hline Categoría Profesional & N & Porcentaje \\
\hline Catedrático Universidad & 14 & $22,6 \%$ \\
Titular Universidad & 26 & $41,9 \%$ \\
Contratado Doctor & 10 & $16,1 \%$ \\
Ayudante doctor & 1 & $1,6 \%$ \\
Asociado & 2 & $3,2 \%$ \\
Técnico Superior & 1 & $1,6 \%$ \\
Total Válidos & 54 & $\mathbf{8 7 , 1} \%$ \\
No Contesta & 8 & $12,9 \%$ \\
Total & $\mathbf{6 2}$ & $\mathbf{1 0 0} \%$ \\
\hline
\end{tabular}


Cuadro 2

Cargo académico de los participantes.

\begin{tabular}{lcc}
\hline \multicolumn{1}{c}{ Cargo académico } & N & Porcentaje \\
\hline Rector & 1 & $1,6 \%$ \\
Vice rector & 3 & $4,8 \%$ \\
Presidente Consejo Social & 1 & $1,6 \%$ \\
Secretario Consejo Social & 6 & $9,7 \%$ \\
Decano & 2 & $3,2 \%$ \\
Vicedecano & 2 & $3,2 \%$ \\
Servicios Jurídicos & 6 & $9,7 \%$ \\
Secretario departamento & 1 & $1,6 \%$ \\
Secretario general & 1 & $1,6 \%$ \\
Vicesecretario general & 1 & $1,6 \%$ \\
Total Válidos & $\mathbf{2 4}$ & $\mathbf{3 8 , 7 \%}$ \\
No Contesta & 38 & $61,3 \%$ \\
Total & $\mathbf{6 2}$ & $\mathbf{1 0 0 \%}$ \\
\hline
\end{tabular}

Cuadro 3

Área de conocimiento de los participantes

\begin{tabular}{lcr}
\hline Área de conocimiento & N & Porcentaje \\
\hline Ciencias Sociales y Jurídicas & 37 & $59,7 \%$ \\
Ingenierías y Arquitectura & 11 & $17,7 \%$ \\
Ciencias de la Salud & 4 & $6,5 \%$ \\
Ciencias & 3 & $4,8 \%$ \\
Artes y Humanidades & 3 & $4,8 \%$ \\
PAS & 3 & $4,8 \%$ \\
Total Válidos & $\mathbf{6 1}$ & $\mathbf{9 8 , 4} \%$ \\
No Contesta & 1 & $\mathbf{1 , 6 \%}$ \\
Total & $\mathbf{6 2}$ & $\mathbf{1 0 0} \%$ \\
\hline
\end{tabular}




\section{Cuestionario y procedimiento}

El cuestionario utilizado se diseñó a partir del análisis bibliográfico de la literatura focalizada en la integridad académica y el fraude del alumnado español en las evaluaciones (Comas, 2009; Comas et al. 2010, Comas et al., 2011; Comas \& Sureda, 2016; Foltynek, 2013; Teixeira \& Rocha, 2006). De acuerdo con los resultados de este análisis se identificaron 29 comportamientos fraudulentos y 10 tipos de sanciones aplicables.

Respecto a los comportamientos, el cuestionario contemplaba dos preguntas. La primera constaba de 29 ítems con los comportamientos fraudulentos del alumnado en las evaluaciones localizados en la literatura (tabla 1). Los participantes tenían que valorar la gravedad de cada comportamiento. La segunda era una pregunta abierta que permitía a los participantes señalar otras acciones fraudulentas. Con las respuestas se obtuvo un inventario de 12 nuevos comportamientos (tabla 2) que fueron valorados por los participantes en una segunda consulta. Cabe señalar que en esta segunda ronda solo respondieron 29 de los 62 participantes iniciales.

Respecto a las sanciones aplicables ante comportamientos fraudulentos, el cuestionario contemplaba tres preguntas: 1) una para faltas consideradas leves (tabla 3), 2) una para faltas consideradas graves (tabla 4); y 3) otra para faltas consideradas muy graves (tabla 5). En cada una de estas preguntas, se proponían 10 ítems con los mismos tipos de sanciones. Los participantes debían señalar, para cada ítem, si estaban en: a) desacuerdo; b) ni acuerdo ni desacuerdo; o c) de acuerdo.

\section{Estrategia de análisis}

Para analizar el grado de acuerdo entre expertos respecto a una serie de ítems de naturaleza categórica nominal se han utilizado tres indicadores diferentes pero complementarios. El primero de ellos son las tablas de frecuencias de las preguntas, que permiten observar las distribuciones así como las categorías que acumulan mayor porcentaje de respuestas. En segundo lugar, se calculó el \% of overal agreement de los expertos respecto a cada ítem y respecto al total de los ítems de cada pregunta. Algunos autores, como Jakobsson \& Westergren (2005), señalan que se trata de una medida insuficiente debido a que no se incluye el acuerdo esperado por el azar. Por eso, y en tercer lugar, se calculó el free-marginal multirater Kappa como medida de la fuerza de la concordancia entre los expertos tanto para cada ítem como para el total de los ítems de cada pregunta.

El free-marginal multirater Kappa (Randolph, 2008; Warrens, 2010), con la variación de la fórmula de Gwet (2010), es una medida de concordancia ajustada al azar, útil cuando se tienen múltiples evaluadores y múltiples categorías nominales. Se trata de una alternativa al fixed-marginal multirater Kappa de Fleiss (1971), que resulta más pertinente cuando los expertos están obligados a asignar un número de casos a cada categoría (Brennan \& Prediger, 1981), cosa que no ocurría en nuestro estudio.

En cuanto a las interpretaciones del índice de Kappa destaca la de Fleiss (1981) que señala que los valores muestran una fuerza de la concordancia regular entre 0.400.60, buena entre 0.61-0.75 y excelente para los valores superiores a 0.75 . Por su parte, Altman (1991) propone una clasificación más amplia de la interpretación donde Kappa 
señalaría una fuerza de la concordancia pobre para valores $<0.20$, débil entre 0.21-0.40, moderada entre 0.41-0.60, buena entre 0.61-0.80 y muy buena entre 0.81-1. Siguiendo esta clasificación se han considerado comportamientos con un consenso aceptable aquellos que presentaban un índice de Kappa de Fleiss superior a 0,40, lo que coincide con las categorías "regular" de Fleis y "moderado" de Altman.

\section{Resultados}

\section{En relación a la pregunta ¿Cuáles son las principales conductas fraudulentas en las evaluaciones del alumnado? \\ Se han identificado 41 comportamientos fraudulentos por parte del alumnado en las evaluaciones: 29 obtenidos del análisis de la literatura (tabla 1) y 12 aportados por el panel de expertos (tabla 2).}

Tabla 1.

Nivel de gravedad otorgado a una serie de acciones fraudulentas en las evaluaciones.

\begin{tabular}{|c|c|c|c|c|c|c|c|}
\hline & $\begin{array}{l}\text { NO ES } \\
\text { FALTA }\end{array}$ & $\begin{array}{l}\text { FALTA } \\
\text { LEVE }\end{array}$ & $\begin{array}{l}\text { FALTA } \\
\text { GRAVE }\end{array}$ & $\begin{array}{l}\text { FALTA } \\
\text { MUY } \\
\text { GRAVE }\end{array}$ & TOTAL & $\begin{array}{c}\% \\
\text { Consenso } \\
\text { General }\end{array}$ & $\begin{array}{c}\text { Free-marginal } \\
\text { Kappa }\end{array}$ \\
\hline \multirow{2}{*}{$\begin{array}{l}\text { p2_6. Suplantar la identidad de otra } \\
\text { persona en una prueba de evaluación }\end{array}$} & 0 & 1 & 3 & 58 & 62 & \multirow[t]{2}{*}{$87,57 \%$} & \multirow[t]{2}{*}{0,83} \\
\hline & $0,0 \%$ & $1,6 \%$ & $4,8 \%$ & $93,5 \%$ & $100 \%$ & & \\
\hline \multirow{2}{*}{$\begin{array}{l}\text { p2_11. Sustraer las pruebas o } \\
\text { exámenes, manipularlos y cambiarlos } \\
\text { por otros }\end{array}$} & 0 & 0 & 6 & 56 & 62 & \multirow[t]{2}{*}{$82,23 \%$} & \multirow[t]{2}{*}{0,76} \\
\hline & $0,0 \%$ & $0,0 \%$ & $9,7 \%$ & $90,3 \%$ & $100 \%$ & & \\
\hline \multirow{2}{*}{$\begin{array}{l}\text { p2_9. Sustraer los enunciados de un } \\
\text { examen u otro tipo de pruebas de } \\
\text { evaluación antes de su realización }\end{array}$} & 0 & 0 & 8 & 54 & 62 & \multirow[t]{2}{*}{$77,15 \%$} & \multirow[t]{2}{*}{0,70} \\
\hline & $0,0 \%$ & $0,0 \%$ & $12,9 \%$ & $87,1 \%$ & $100 \%$ & & \\
\hline \multirow{2}{*}{$\begin{array}{l}\text { p2_7. Presentar como propio un exa- } \\
\text { men realizado por otro alumno }\end{array}$} & 0 & 1 & 7 & 54 & 62 & \multirow[t]{2}{*}{$76,78 \%$} & \multirow[t]{2}{*}{0,69} \\
\hline & $0,0 \%$ & $1,6 \%$ & $11,3 \%$ & $87,1 \%$ & $100 \%$ & & \\
\hline \multirow{2}{*}{$\begin{array}{l}\text { p2_5. Copiar en un examen pres- } \\
\text { encial a través de dispositivos tec- } \\
\text { nológicos (teléfonos, pinganillos, etc.) }\end{array}$} & 0 & 1 & 14 & 47 & 62 & \multirow[t]{2}{*}{$61,98 \%$} & \multirow[t]{2}{*}{0,49} \\
\hline & $0,0 \%$ & $1,6 \%$ & $22,6 \%$ & $75,8 \%$ & $100 \%$ & & \\
\hline \multirow{2}{*}{$\begin{array}{l}\text { p2_24. Presentar como propio un } \\
\text { trabajo de otro alumno (atribuirse la } \\
\text { realización de trabajos ajenos) }\end{array}$} & 0 & 3 & 15 & 44 & 62 & \multirow[t]{2}{*}{$57,74 \%$} & \multirow[t]{2}{*}{0,41} \\
\hline & $0,0 \%$ & $4,8 \%$ & $24,2 \%$ & $71,0 \%$ & $100 \%$ & & \\
\hline \multirow{2}{*}{$\begin{array}{l}\text { p2_26. Presentar como propio un } \\
\text { trabajo "comprado" }\end{array}$} & 0 & 4 & 16 & 42 & 62 & \multirow[t]{2}{*}{$52,19 \%$} & \multirow[t]{2}{*}{0,36} \\
\hline & $0,0 \%$ & $6,5 \%$ & $25,8 \%$ & $67,7 \%$ & $100 \%$ & & \\
\hline \multirow{2}{*}{$\begin{array}{l}\text { p2_10. Sustraer exámenes u otros } \\
\text { tipos de pruebas de evaluación una } \\
\text { vez realizados }\end{array}$} & 2 & 5 & 14 & 41 & 62 & \multirow[t]{2}{*}{$48,76 \%$} & \multirow[t]{2}{*}{0,32} \\
\hline & $3,2 \%$ & $8,1 \%$ & $22,6 \%$ & $66,1 \%$ & $100 \%$ & & \\
\hline
\end{tabular}




\begin{tabular}{|c|c|c|c|c|c|c|c|}
\hline & $\begin{array}{l}\text { NO ES } \\
\text { FALTA }\end{array}$ & $\begin{array}{l}\text { FALTA } \\
\text { LEVE }\end{array}$ & $\begin{array}{l}\text { FALTA } \\
\text { GRAVE }\end{array}$ & $\begin{array}{l}\text { FALTA } \\
\text { MUY } \\
\text { GRAVE }\end{array}$ & TOTAL & $\begin{array}{c}\% \\
\text { Consenso } \\
\text { General }\end{array}$ & $\begin{array}{c}\text { Free-marginal } \\
\text { Kappa }\end{array}$ \\
\hline \multirow{2}{*}{$\begin{array}{l}\text { p2_29. Entregar un trabajo que pre- } \\
\text { senta más de un } 50 \% \text { de fragmentos } \\
\text { plagiados }\end{array}$} & 0 & 4 & 19 & 39 & 62 & $48,55 \%$ & 0,31 \\
\hline & $0,0 \%$ & $6,5 \%$ & $30,6 \%$ & $62,9 \%$ & $100 \%$ & & \\
\hline \multirow{2}{*}{$\begin{array}{l}\text { p2_3. Copiar durante un examen } \\
\text { presencial de apuntes, libros o mate- } \\
\text { riales no autorizados }\end{array}$} & 1 & 3 & 36 & 22 & 62 & $45,69 \%$ & 0,28 \\
\hline & $1,6 \%$ & $4,8 \%$ & $58,1 \%$ & $35,5 \%$ & $100 \%$ & & \\
\hline \multirow{2}{*}{$\begin{array}{l}\text { p2_25. Presentar como propio un } \\
\text { trabajo que el alumno/a se ha descar- } \\
\text { gado de un portal de internet (tipo El } \\
\text { Rincón del Vago, monografías.com, } \\
\text { patatabrava.com) }\end{array}$} & 0 & 5 & 23 & 34 & 62 & $43,57 \%$ & 0,25 \\
\hline & $0,0 \%$ & $8,1 \%$ & $37,1 \%$ & $54,8 \%$ & $100 \%$ & & \\
\hline \multirow{2}{*}{$\begin{array}{l}\text { p2_2. Copiar en un examen presen- } \\
\text { cial mediante "chuletas" }\end{array}$} & 1 & 5 & 35 & 21 & 62 & $43,10 \%$ & 0,24 \\
\hline & $1,6 \%$ & $8,1 \%$ & $56,5 \%$ & $33,9 \%$ & $100 \%$ & & \\
\hline \multirow{2}{*}{$\begin{array}{l}\text { p2_20. Incluir datos falsos en un } \\
\text { trabajo }\end{array}$} & 1 & 9 & 16 & 36 & 62 & $41,57 \%$ & 0,22 \\
\hline & $1,6 \%$ & $14,5 \%$ & $25,8 \%$ & $58,1 \%$ & $100 \%$ & & \\
\hline \multirow{2}{*}{$\begin{array}{l}\text { p2_4. Copiar de otro estudiante en } \\
\text { un examen }\end{array}$} & 0 & 8 & 34 & 20 & 62 & $41,20 \%$ & 0,22 \\
\hline & $0,0 \%$ & $12,9 \%$ & $54,8 \%$ & $32,3 \%$ & $100 \%$ & & \\
\hline \multirow[b]{2}{*}{$\begin{array}{l}\text { p2_1. Comunicarse, por cualquier } \\
\text { medio, con un compañero que esté } \\
\text { dentro o fuera del aula durante la } \\
\text { realización de exámenes presenciales } \\
\text { con el fin de obtener información/ } \\
\text { ayuda para contestar las preguntas } \\
\text { de la prueba }\end{array}$} & 1 & 7 & 25 & 29 & 62 & $38,45 \%$ & 0,18 \\
\hline & $1,6 \%$ & $11,3 \%$ & $40,3 \%$ & $46,8 \%$ & $100 \%$ & & \\
\hline \multirow{2}{*}{$\begin{array}{l}\text { p2_28. Entregar un trabajo que } \\
\text { presenta entre un } 25 \% \text { y un } 50 \% \text { de } \\
\text { fragmentos plagiados }\end{array}$} & 0 & 11 & 32 & 19 & 62 & $38,18 \%$ & 0,18 \\
\hline & $0,0 \%$ & $17,7 \%$ & $51,6 \%$ & $30,6 \%$ & $100 \%$ & & \\
\hline \multirow[t]{2}{*}{ p2_15. Citar de forma incorrecta } & 26 & 28 & 8 & 0 & 62 & $38,66 \%$ & 0,18 \\
\hline & $41,9 \%$ & $45,2 \%$ & $12,9 \%$ & $0,0 \%$ & $100 \%$ & & \\
\hline \multirow{2}{*}{$\begin{array}{l}\text { p2_8. Colaborar, encubrir o favorecer } \\
\text { la copia durante un examen }\end{array}$} & 3 & 15 & 34 & 10 & 62 & $37,76 \%$ & 0,17 \\
\hline & $4,8 \%$ & $24,2 \%$ & $54,8 \%$ & $16,1 \%$ & $100 \%$ & & \\
\hline \multirow{2}{*}{$\begin{array}{l}\text { p2_13. Presentar un trabajo con copia } \\
\text { literal de textos sin citar su proce- } \\
\text { dencia }\end{array}$} & 3 & 10 & 34 & 15 & 62 & $37,76 \%$ & 0,17 \\
\hline & $4,8 \%$ & $16,1 \%$ & $54,8 \%$ & $24,2 \%$ & $100 \%$ & & \\
\hline \multirow{2}{*}{$\begin{array}{l}\text { p2_27. Entregar un trabajo que pre- } \\
\text { senta menos de un } 25 \% \text { de fragmen- } \\
\text { tos plagiados }\end{array}$} & 4 & 31 & 19 & 8 & 62 & $35,43 \%$ & 0,14 \\
\hline & $6,5 \%$ & $50,0 \%$ & $30,6 \%$ & $12,9 \%$ & $100 \%$ & & \\
\hline \multirow{2}{*}{$\begin{array}{l}\text { p2_18. Inventarse las citas y/o las } \\
\text { referencias bibliográficas }\end{array}$} & 2 & 10 & 23 & 27 & 62 & $34,37 \%$ & 0,12 \\
\hline & $3,2 \%$ & $16,1 \%$ & $37,1 \%$ & $43,5 \%$ & $100 \%$ & & \\
\hline \multirow{2}{*}{$\begin{array}{l}\text { p2_14. Presentar un trabajo con citas } \\
\text { indirectas (paráfrasis) sin citar las } \\
\text { fuentes }\end{array}$} & 8 & 20 & 29 & 5 & 62 & $33,53 \%$ & 0,11 \\
\hline & $12,9 \%$ & $32,3 \%$ & $46,8 \%$ & $8,1 \%$ & $100 \%$ & & \\
\hline
\end{tabular}




\begin{tabular}{|c|c|c|c|c|c|c|c|}
\hline & $\begin{array}{l}\text { NO ES } \\
\text { FALTA }\end{array}$ & $\begin{array}{l}\text { FALTA } \\
\text { LEVE }\end{array}$ & $\begin{array}{l}\text { FALTA } \\
\text { GRAVE }\end{array}$ & $\begin{array}{l}\text { FALTA } \\
\text { MUY } \\
\text { GRAVE }\end{array}$ & TOTAL & $\begin{array}{c}\% \\
\text { Consenso } \\
\text { General }\end{array}$ & $\begin{array}{c}\text { Free-marginal } \\
\text { Kappa }\end{array}$ \\
\hline \multirow{2}{*}{$\begin{array}{l}\text { p2_21. Menospreciar el aporte de una } \\
\text { persona en el trabajo }\end{array}$} & 5 & 19 & 29 & 9 & 62 & $32,95 \%$ & 0,11 \\
\hline & $8,1 \%$ & $30,6 \%$ & $46,8 \%$ & $14,5 \%$ & $100 \%$ & & \\
\hline \multirow{2}{*}{$\begin{array}{l}\text { p2_12. No poner en conocimiento } \\
\text { del profesorado casos conocidos de } \\
\text { fraude cometidos por otros alumnos }\end{array}$} & 20 & 27 & 13 & 2 & 62 & $32,79 \%$ & 0,10 \\
\hline & $32,3 \%$ & $43,5 \%$ & $21,0 \%$ & $3,2 \%$ & $100 \%$ & & \\
\hline \multirow{2}{*}{$\begin{array}{l}\text { p2_22. Exagerar el aporte de una per- } \\
\text { sona en un trabajo con el objetivo de } \\
\text { que el profesor crea que es de autoría } \\
\text { compartida }\end{array}$} & 5 & 24 & 25 & 8 & 62 & $32,47 \%$ & 0,10 \\
\hline & $8,1 \%$ & $38,7 \%$ & $40,3 \%$ & $12,9 \%$ & $100 \%$ & & \\
\hline \multirow{2}{*}{$\begin{array}{l}\text { p2_17. Falsificar datos de las referen- } \\
\text { cias bibliográficas }\end{array}$} & 1 & 15 & 22 & 24 & 62 & $32,36 \%$ & 0,10 \\
\hline & $1,6 \%$ & $24,2 \%$ & $35,5 \%$ & $38,7 \%$ & $100 \%$ & & \\
\hline \multirow{2}{*}{$\begin{array}{l}\text { p2_23. Presentar un trabajo propio } \\
\text { ya evaluado en otra asignatura o en } \\
\text { otro curso }\end{array}$} & 5 & 9 & 26 & 22 & 62 & $31,84 \%$ & 0,09 \\
\hline & $8,1 \%$ & $14,5 \%$ & $41,9 \%$ & $35,5 \%$ & $100 \%$ & & \\
\hline \multirow{2}{*}{$\begin{array}{l}\text { p2_19. Suprimir datos relevantes en } \\
\text { un trabajo }\end{array}$} & 8 & 17 & 22 & 15 & 62 & $26,44 \%$ & 0,02 \\
\hline & $12,9 \%$ & $27,4 \%$ & $35,5 \%$ & $24,2 \%$ & $100 \%$ & & \\
\hline \multirow{2}{*}{$\begin{array}{l}\text { p2_16. No incluir las referencias } \\
\text { bibliográficas de los documentos } \\
\text { utilizados }\end{array}$} & 17 & 24 & 17 & 4 & 62 & $29,30 \%$ & 0,01 \\
\hline & $27,4 \%$ & $38,7 \%$ & $27,4 \%$ & $6,5 \%$ & $100 \%$ & & \\
\hline TOTAL ÍTEMS & & & & & & $45,46 \%$ & 0,27 \\
\hline
\end{tabular}

95\% Intervalo de confianza [0,19; 0,35]

\section{Tabla 2}

Nivel de gravedad otorgado a una serie de acciones deshonestas en las evaluaciones. Ítems propuestos por los participantes

\begin{tabular}{|c|c|c|c|c|c|c|c|}
\hline & $\begin{array}{l}\text { NO ES } \\
\text { FALTA }\end{array}$ & $\begin{array}{l}\text { FALTA } \\
\text { LEVE }\end{array}$ & $\begin{array}{l}\text { FALTA } \\
\text { GRAVE }\end{array}$ & $\begin{array}{l}\text { FALTA } \\
\text { MUY } \\
\text { GRAVE }\end{array}$ & TOTAL & $\begin{array}{c}\% \\
\text { Consenso } \\
\text { General }\end{array}$ & $\begin{array}{c}\text { Free-marginal } \\
\text { Kappa }\end{array}$ \\
\hline \multirow{2}{*}{$\begin{array}{l}\text { 3.3. Acceder por cualquier medio a la } \\
\text { base de datos de las calificaciones y } \\
\text { modificar las notas/actas. }\end{array}$} & 0 & 0 & 1 & 28 & 29 & \multirow[t]{2}{*}{$93,10 \%$} & \multirow[t]{2}{*}{0,91} \\
\hline & $0,0 \%$ & $0,0 \%$ & $3,4 \%$ & $96,6 \%$ & $100 \%$ & & \\
\hline \multirow{2}{*}{$\begin{array}{l}\text { 3.10. Falsificación documental de } \\
\text { documentos que pueden suponer } \\
\text { convalidar un examen o una prueba. }\end{array}$} & 0 & 0 & 2 & 27 & 29 & \multirow[t]{2}{*}{$86,70 \%$} & \multirow[t]{2}{*}{0,82} \\
\hline & $0,0 \%$ & $0,0 \%$ & $6,9 \%$ & $93,1 \%$ & $100 \%$ & & \\
\hline \multirow{2}{*}{$\begin{array}{l}\text { 3.11. Usurpar identidad online de un } \\
\text { compañero. }\end{array}$} & 0 & 0 & 3 & 26 & 29 & \multirow[t]{2}{*}{$80,79 \%$} & \multirow[t]{2}{*}{0,74} \\
\hline & $0,0 \%$ & $0,0 \%$ & $10,3 \%$ & $89,7 \%$ & $100 \%$ & & \\
\hline \multirow{2}{*}{$\begin{array}{l}\text { 3.6. Aparentar que el profesor ha } \\
\text { perdido el examen cuando en reali- } \\
\text { dad no lo entregó. }\end{array}$} & 1 & 1 & 7 & 20 & 29 & \multirow[t]{2}{*}{$51,97 \%$} & \multirow[t]{2}{*}{0,36} \\
\hline & $3,4 \%$ & $3,4 \%$ & $24,1 \%$ & $69,0 \%$ & $100 \%$ & & \\
\hline
\end{tabular}




\begin{tabular}{|c|c|c|c|c|c|c|c|}
\hline & $\begin{array}{l}\text { NO ES } \\
\text { FALTA }\end{array}$ & $\begin{array}{l}\text { FALTA } \\
\text { LEVE }\end{array}$ & $\begin{array}{l}\text { FALTA } \\
\text { GRAVE }\end{array}$ & $\begin{array}{l}\text { FALTA } \\
\text { MUY } \\
\text { GRAVE }\end{array}$ & TOTAL & $\begin{array}{c}\% \\
\text { Consenso } \\
\text { General }\end{array}$ & $\begin{array}{c}\text { Free-marginal } \\
\text { Kappa }\end{array}$ \\
\hline \multirow{2}{*}{$\begin{array}{l}\text { 3.4. Simular que se ha realizado una } \\
\text { prueba de evaluación cuando en } \\
\text { realidad no se ha hecho. }\end{array}$} & 0 & 1 & 9 & 19 & 29 & \multirow[t]{2}{*}{$50,99 \%$} & \multirow[t]{2}{*}{0,35} \\
\hline & $0,0 \%$ & $3,4 \%$ & $31,0 \%$ & $65,5 \%$ & $100 \%$ & & \\
\hline \multirow{2}{*}{$\begin{array}{l}\text { 3.5. Modificar una evaluación du- } \\
\text { rante la revisión. }\end{array}$} & 2 & 0 & 8 & 19 & 29 & \multirow[t]{2}{*}{$49,26 \%$} & \multirow[t]{2}{*}{0,32} \\
\hline & $6,9 \%$ & $0,0 \%$ & $27,6 \%$ & $65,5 \%$ & $100 \%$ & & \\
\hline \multirow{2}{*}{$\begin{array}{l}\text { 3.8. Incluir a un compañero en un } \\
\text { trabajo en cuya elaboración no ha } \\
\text { participado. }\end{array}$} & 1 & 6 & 19 & 3 & 29 & \multirow[t]{2}{*}{$46,55 \%$} & \multirow[t]{2}{*}{0,29} \\
\hline & $3,4 \%$ & $20,7 \%$ & $65,5 \%$ & $10,3 \%$ & $100 \%$ & & \\
\hline \multirow{2}{*}{$\begin{array}{l}\text { 3.12. Negar los hechos de fraude } \\
\text { cuando son "in fraganti". }\end{array}$} & 2 & 2 & 12 & 13 & 29 & \multirow[t]{2}{*}{$35,96 \%$} & \multirow[t]{2}{*}{0,15} \\
\hline & $6,9 \%$ & $6,9 \%$ & $41,4 \%$ & $44,8 \%$ & $100 \%$ & & \\
\hline \multirow{2}{*}{$\begin{array}{l}\text { 3.7. No seguir la instrucción de un } \\
\text { profesor de desprenderse de dis- } \\
\text { positivos electrónicos durante una } \\
\text { prueba de evaluación. }\end{array}$} & 1 & 4 & 14 & 10 & 29 & \multirow[t]{2}{*}{$34,98 \%$} & \multirow[t]{2}{*}{0,13} \\
\hline & $3,4 \%$ & $13,8 \%$ & $48,3 \%$ & $34,5 \%$ & $100 \%$ & & \\
\hline \multirow{2}{*}{$\begin{array}{l}\text { 3.1. Hostigar a un compañero para } \\
\text { que se deje copiar (en al aula o } \\
\text { fuera). }\end{array}$} & 1 & 4 & 12 & 12 & 29 & \multirow[t]{2}{*}{$33,99 \%$} & \multirow[t]{2}{*}{0,12} \\
\hline & $3,4 \%$ & $13,8 \%$ & $41,4 \%$ & $41,4 \%$ & $100 \%$ & & \\
\hline \multirow{2}{*}{$\begin{array}{l}\text { 3.9. Presentar como propias prácticas } \\
\text { oficiales realizadas en academias } \\
\text { privadas. }\end{array}$} & 2 & 5 & 15 & 7 & 29 & \multirow[t]{2}{*}{$33,74 \%$} & \multirow[t]{2}{*}{0,12} \\
\hline & $6,9 \%$ & $17,2 \%$ & $51,7 \%$ & $24,1 \%$ & $100 \%$ & & \\
\hline \multirow{2}{*}{$\begin{array}{l}\text { 3.2. Sacar, por cualquier medio, el } \\
\text { enunciado de un examen fuera del } \\
\text { aula cuando el profesor lo había } \\
\text { prohibido. }\end{array}$} & 0 & 8 & 10 & 11 & 29 & \multirow[t]{2}{*}{$31,53 \%$} & \multirow[t]{2}{*}{0,09} \\
\hline & $0,0 \%$ & $27,6 \%$ & $34,5 \%$ & $37,9 \%$ & $100 \%$ & & \\
\hline TOTAL ÍTEMS & & & & & & $52,46 \%$ & 0,37 \\
\hline
\end{tabular}

$95 \%$ Intervalo de confianza $[0,20,0,53]$

\section{En relación a la pregunta ¿Cuál es el nivel de gravedad de cada uno de estos comportamientos fraudulentos?}

Por lo que respecta a la valoración de los 29 comportamientos fraudulentos contemplados en el cuestionario y obtenidos del análisis de la literatura (tabla 1), de forma global se observa un $45,46 \%$ de consenso general entre jueces y una fuerza de la concordancia más bien débil $(\mathrm{K}=0.27)$.

Analizando individualmente los indicadores de los ítems, se observa que: 1 ítem presenta una concordancia muy buena o excelente $(87,57 \%$ de consenso general y $\mathrm{K}=0.83$ ); 3 ítems presentan una concordancia buena (más del $76 \%$ de consenso general y Kappas superiores a 0.68); 2 ítems presentan una concordancia moderada o regular (más del 57\% de consenso general y Kappas superiores a 0.40); 8 ítems presentan una concordancia débil (menos del 53\% de consenso general y Kappas menores de 0.37); y los 15 ítems restantes presentan una concordancia pobre (menos del 39\% de consenso general y Kappas menores de 0.19).

Respecto a la clasificación de los 12 comportamientos fraudulentos aportados por los expertos durante la primera ronda (tabla 2), de forma global se observa un $52 \%$ de consenso general y una fuerza de la concordancia entre jueces débil ( $\mathrm{K}=0.37)$. 
Analizando individualmente los indicadores de los ítems se observa que: 2 ítems presentan una concordancia muy buena o excelente (más del $86 \%$ de consenso general y kappas superiores a 0.81$)$; 1 ítem presenta una concordancia buena (80,79\% de consenso general y Kappa=0.74); 4 ítems presentan una concordancia débil (entre 51,97\% y el 46,55\% de consenso general y un Kappas menores de 0.37); y los 5 ítems restantes presentan una concordancia pobre (menos del 40\% de consenso general y Kappas menores de 0.2).

\section{Clasificación de los comportamientos fraudulentos.}

A partir de los resultados obtenidos, tan solo 9 de los 41 comportamientos fraudulentos analizados se clasificaron como "muy graves" con una concordancia moderada o superior entre los expertos, es decir, con un índice de Kappa de Fleiss mayor que 0,40 (cuadro 4).

Por el contrario, el resto de los 32 comportamientos fraudulentos analizados presentaban una concordancia débil o pobre (Kappa de Fleiss $<0,40$ ), por lo que no se pudieron clasificar en ninguna categoría. No obstante, vale la pena señalar que, en la mayoría de casos, las divergencias a la hora de clasificar los comportamientos se encontraban entre las categorías grave y muy grave (ver tablas 1 y 2).

Cuadro 4

Acciones fraudulentas consideradas muy graves (Kappa de Fleiss $>0,40$ )

\section{Contemplados en el cuestionario}

Suplantar la identidad de otra persona en una prueba de evaluación.

Sustraer las pruebas o exámenes, manipularlos y cambiarlos por otros.

Sustraer los enunciados de un examen u otro tipo de pruebas de evaluación antes de su realización.

Presentar como propio un examen realizado por otro alumno.

Copiar en un examen presencial a través de dispositivos tecnológicos (teléfonos, pinganillos, etc.).

Presentar como propio un trabajo de otro alumno (atribuirse la realización de trabajos ajenos).

\section{Aportados por el panel de expertos}

Acceder por cualquier medio a la base de datos de las calificaciones y modificar las notas/actas.

Falsificación documental (ejemplos: certificados de nivel de idioma, certificados de notas, etc.) de documentos que pueden suponer convalidar un examen o una prueba.

Usurpar identidad online de un compañero.

\footnotetext{
En relación a la pregunta ¿Qué sanciones habría que aplicar a las faltas según su nivel de gravedad?

Sanciones para faltas consideradas leves (tabla 3).
} 
Respecto a las sanciones aplicables a las faltas consideradas leves, de forma general se observa un 56,65\% de consenso general entre jueces del 56,65\% y una fuerza de la concordancia entre jueces débil $(\mathrm{K}=0.35) .2$ ítems presentan una concordancia buena (más del 73\% de consenso general y Kappas superiores a 0.60); 1 ítem presenta una concordancia moderada (71,23\% de consenso general y Kappa=0.57); 4 ítems presentan una concordancia débil (menos del 60\% de consenso general y Kappas por debajo de 0.4 ); y 3 ítems presentan una concordancia pobre (Menos del 50\% de consenso general y Kappas inferiores a 0.2).

Las sanciones con un nivel de concordancia entre los expertos aceptable (Kappa de Fleiss > 0,40) fueron tres: a) "p5_a_9. Expulsión de la universidad" (K=0,69); b) "p5_a_4. Suspender todas las asignaturas matriculadas en este año académico" (K=,61); y c) "p5_a_8. Prohibición temporal de acceso a la universidad" (K=0,57). No obstante, en las tres sanciones, el consenso se situaba en la categoría de respuesta "en desacuerdo". Es decir, el consenso de los expertos estaba en considerar estas tres sanciones como excesivas para aplicarlas ante faltas leves.

Por otro lado, la sanción con mayor grado de concordancia entre los expertos para aplicarse ante faltas leves fue la "Amonestación verbal", con un porcentaje de respuesta del 69,35\% pero con una concordancia débil $(\mathrm{K}=0,29)$.

Tabla 3.

Sanciones aplicables a faltas consideradas leves.

\begin{tabular}{|c|c|c|c|c|c|c|}
\hline & Desacuerdo & Neutralidad & Consenso & Total & $\begin{array}{c}\% \\
\text { Consenso } \\
\text { general }\end{array}$ & $\begin{array}{c}\text { Free-marginal } \\
\text { Kappa }\end{array}$ \\
\hline \multirow{2}{*}{$\begin{array}{l}\text { Expulsión de la } \\
\text { universidad ante faltas leves }\end{array}$} & 55 & 3 & 4 & 62 & \multirow[t]{2}{*}{$79,01 \%$} & \multirow[t]{2}{*}{0,69} \\
\hline & $88,71 \%$ & $4,84 \%$ & $6,45 \%$ & $100 \%$ & & \\
\hline \multirow{2}{*}{$\begin{array}{l}\text { Suspender todas las asignaturas } \\
\text { matriculadas en este año aca- } \\
\text { démico ante faltas leves }\end{array}$} & 53 & 4 & 5 & 62 & \multirow[t]{2}{*}{$73,72 \%$} & \multirow[t]{2}{*}{0,61} \\
\hline & $85,48 \%$ & $6,45 \%$ & $8,06 \%$ & $100 \%$ & & \\
\hline \multirow{2}{*}{$\begin{array}{l}\text { Prohibición temporal de acceso } \\
\text { a la universidad ante faltas } \\
\text { leves }\end{array}$} & 52 & 4 & 6 & 62 & \multirow[t]{2}{*}{$71,23 \%$} & \multirow[t]{2}{*}{0,57} \\
\hline & $83,87 \%$ & $6,45 \%$ & $9,68 \%$ & $100 \%$ & & \\
\hline \multirow{2}{*}{$\begin{array}{l}\text { Revisar evaluaciones anteriores } \\
\text { ante faltas leves }\end{array}$} & 47 & 9 & 6 & 62 & \multirow[t]{2}{*}{$59,86 \%$} & \multirow[t]{2}{*}{0,40} \\
\hline & $75,81 \%$ & $14,52 \%$ & $9,68 \%$ & $100 \%$ & & \\
\hline \multirow{2}{*}{$\begin{array}{l}\text { Pérdida renovación de becas o } \\
\text { ayudas propias de la universi- } \\
\text { dad ante faltas leves }\end{array}$} & 46 & 9 & 7 & 62 & \multirow[t]{2}{*}{$57,75 \%$} & \multirow[t]{2}{*}{0,37} \\
\hline & $74,19 \%$ & $14,52 \%$ & $11,29 \%$ & $100 \%$ & & \\
\hline \multirow{2}{*}{$\begin{array}{l}\text { Pérdida de derechos de gratui- } \\
\text { dad de créditos ante faltas leves }\end{array}$} & 44 & 8 & 10 & 62 & \multirow[t]{2}{*}{$53,89 \%$} & \multirow[t]{2}{*}{0,31} \\
\hline & $70,97 \%$ & $12,90 \%$ & $16,13 \%$ & $100 \%$ & & \\
\hline \multirow{2}{*}{$\begin{array}{l}\text { Amonestación verbal ante faltas } \\
\text { leves }\end{array}$} & 12 & 7 & 43 & 62 & \multirow[t]{2}{*}{$52,35 \%$} & \multirow[t]{2}{*}{0,29} \\
\hline & $19,35 \%$ & $11,29 \%$ & $69,35 \%$ & $100 \%$ & & \\
\hline \multirow{2}{*}{$\begin{array}{l}\text { Suspender la asignatura en este } \\
\text { año académico ante faltas leves }\end{array}$} & 39 & 12 & 11 & 62 & \multirow[t]{2}{*}{$45,58 \%$} & \multirow[t]{2}{*}{0,18} \\
\hline & $62,90 \%$ & $19,35 \%$ & $17,74 \%$ & $100 \%$ & & \\
\hline
\end{tabular}




\begin{tabular}{|c|c|c|c|c|c|c|}
\hline & Desacuerdo & Neutralidad & Consenso & Total & $\begin{array}{c}\% \\
\text { Consenso } \\
\text { general }\end{array}$ & $\begin{array}{c}\text { Free-marginal } \\
\text { Kappa }\end{array}$ \\
\hline $\begin{array}{l}\text { Pérdida de derecho del } \\
\text { alumno/a infractor a ostentar } \\
\text { ningún cargo de representación } \\
\text { en la universidad ante faltas } \\
\text { leves }\end{array}$ & $50,00 \%$ & $17,74 \%$ & $32,26 \%$ & $100 \%$ & $37,55 \%$ & 0,06 \\
\hline $\begin{array}{l}\text { Suspender acto/prueba de } \\
\text { evaluación ante faltas leves }\end{array}$ & $\begin{array}{c}25 \\
40,32 \%\end{array}$ & $\begin{array}{c}13 \\
20,97 \%\end{array}$ & $\begin{array}{c}24 \\
38,71 \%\end{array}$ & $\begin{array}{c}62 \\
100 \%\end{array}$ & $34,58 \%$ & 0,02 \\
\hline TOTAL ÍTEMS & & & & & $56,65 \%$ & 0,35 \\
\hline
\end{tabular}

95\% Intervalo de confianza [0,21, 0,49]

\section{Sanciones para faltas consideradas graves (tabla 4).}

En relación a las faltas graves se obtiene un $46,52 \%$ de consenso general entre jueces y una fuerza de concordancia entre jueces pobre (Kappa=0.20). 1 ítem presenta una concordancia buena (73,82\% de consenso general y Kappa=0.61); 4 ítems presentan una concordancia débil (menos del $51 \%$ de consenso general y Kappas inferiores a 0.27); 5 ítems presentan una concordancia pobre (menos del $43 \%$ de consenso general y Kappas inferiores a 0.15).

La única sanción para faltas graves con un nivel suficiente de concordancia (Kappa de Fleiss > 0,40) fue: "p5_b_2. Suspender acto/prueba de evaluación" (K=0.61).

Las otras dos sanciones aplicables ante faltas graves con mayor porcentaje de respuesta fueron: a) "p5_b_7. Pérdida de derecho del alumno/a infractor a ostentar ningún cargo de representación en la universidad" con un porcentaje de respuesta del $67,74 \%$ pero una concordancia débil $(\mathrm{K}=0,26)$; y b) "p5_b_3. Suspender la asignatura en este año académico" con un porcentaje de respuesta del $66,13 \%$ pero una concordancia también débil $(K=0,23)$.

Tabla 4

Sanciones aplicables a faltas consideradas graves

\begin{tabular}{|c|c|c|c|c|c|c|}
\hline & Desacuerdo & Neutralidad & Consenso & Total & $\begin{array}{c}\% \text { Consenso } \\
\text { General }\end{array}$ & $\begin{array}{c}\text { Free-marginal } \\
\text { Kappa }\end{array}$ \\
\hline \multirow{2}{*}{$\begin{array}{l}\text { Suspender acto/prueba de } \\
\text { evaluación ante faltas graves }\end{array}$} & 3 & 6 & 53 & 62 & \multirow[t]{2}{*}{$73,82 \%$} & \multirow[t]{2}{*}{0,61} \\
\hline & $4,84 \%$ & $9,68 \%$ & $85,48 \%$ & $100 \%$ & & \\
\hline \multirow{2}{*}{$\begin{array}{l}\text { Pérdida de derecho del } \\
\text { alumno/a infractor a ostentar } \\
\text { ningún cargo de representación } \\
\text { en la universidad ante faltas } \\
\text { graves }\end{array}$} & 13 & 7 & 42 & 62 & \multirow[t]{2}{*}{$50,77 \%$} & \multirow[t]{2}{*}{0,26} \\
\hline & $20,97 \%$ & $11,29 \%$ & $67,74 \%$ & $100 \%$ & & \\
\hline \multirow{2}{*}{$\begin{array}{l}\text { Expulsión de la universidad } \\
\text { ante faltas graves }\end{array}$} & 42 & 7 & 13 & 62 & \multirow[t]{2}{*}{$50,77 \%$} & \multirow[t]{2}{*}{0,26} \\
\hline & $67,74 \%$ & $11,29 \%$ & $20,97 \%$ & $100 \%$ & & \\
\hline \multirow{2}{*}{$\begin{array}{l}\text { Suspender la asignatura en } \\
\text { este año académico ante faltas } \\
\text { graves }\end{array}$} & 8 & 13 & 41 & 62 & \multirow[t]{2}{*}{$48,97 \%$} & \multirow[t]{2}{*}{0,23} \\
\hline & $12,90 \%$ & $20,97 \%$ & $66,13 \%$ & $100 \%$ & & \\
\hline
\end{tabular}




\begin{tabular}{|c|c|c|c|c|c|c|}
\hline & Desacuerdo & Neutralidad & Consenso & Total & $\begin{array}{l}\% \text { Consenso } \\
\text { General }\end{array}$ & $\begin{array}{c}\text { Free-marginal } \\
\text { Kappa }\end{array}$ \\
\hline \multirow{2}{*}{$\begin{array}{l}\text { Prohibición temporal de acceso } \\
\text { a la universidad ante faltas } \\
\text { graves }\end{array}$} & 40 & 6 & 16 & 62 & \multirow[t]{2}{*}{$48,39 \%$} & \multirow[t]{2}{*}{0,23} \\
\hline & $64,52 \%$ & $9,68 \%$ & $25,81 \%$ & $100 \%$ & & \\
\hline \multirow{2}{*}{$\begin{array}{l}\text { Suspender todas las asigna- } \\
\text { turas matriculadas en este año } \\
\text { académico ante faltas graves }\end{array}$} & 37 & 11 & 14 & 62 & \multirow[t]{2}{*}{$42,94 \%$} & \multirow[t]{2}{*}{0,14} \\
\hline & $59,68 \%$ & $17,74 \%$ & $22,58 \%$ & $100 \%$ & & \\
\hline \multirow{2}{*}{$\begin{array}{l}\text { Revisar evaluaciones anteriores } \\
\text { ante faltas graves }\end{array}$} & 35 & 10 & 17 & 62 & \multirow[t]{2}{*}{$41,04 \%$} & \multirow[t]{2}{*}{0,12} \\
\hline & $56,45 \%$ & $16,13 \%$ & $27,42 \%$ & $100 \%$ & & \\
\hline \multirow{2}{*}{$\begin{array}{l}\text { Amonestación verbal ante } \\
\text { faltas graves }\end{array}$} & 25 & 8 & 29 & 62 & \multirow[t]{2}{*}{$38,82 \%$} & \multirow[t]{2}{*}{0,08} \\
\hline & $40,32 \%$ & $12,90 \%$ & $46,77 \%$ & $100 \%$ & & \\
\hline \multirow{2}{*}{$\begin{array}{l}\text { Pérdida de derechos de gra- } \\
\text { tuidad de créditos ante faltas } \\
\text { graves }\end{array}$} & 22 & 12 & 28 & 62 & \multirow[t]{2}{*}{$35,70 \%$} & \multirow[t]{2}{*}{0,04} \\
\hline & $35,48 \%$ & $19,35 \%$ & $45,16 \%$ & $100 \%$ & & \\
\hline \multirow{2}{*}{$\begin{array}{l}\text { Pérdida renovación de becas o } \\
\text { ayudas propias de la universi- } \\
\text { dad ante faltas graves }\end{array}$} & 19 & 16 & 27 & 62 & \multirow[t]{2}{*}{$33,95 \%$} & \multirow[t]{2}{*}{0,01} \\
\hline & $30,65 \%$ & $25,81 \%$ & $43,55 \%$ & $100 \%$ & & \\
\hline TOTAL ÍTEMS & & & & & $46,52 \%$ & 0,20 \\
\hline
\end{tabular}

$95 \%$ Intervalo de confianza $[0.09,0.30]$

\section{Sanciones para faltas consideradas muy graves (tabla 5).}

En relación a las faltas muy graves se observa un 53,84\% de consenso general entre jueces y una fuerza de concordancia entre jueces débil (Kappa=0.31). 1 ítem presenta una concordancia muy buena o excelente $(87,57 \%$ de consenso general y Kappa=0.81); 1 ítem presenta una concordancia buena $(79,01 \%$ de consenso general y Kappa=0.69); 1 ítem presenta una concordancia moderada (64,20\% de consenso general y Kappa=0.46); 2 ítems presentan una concordancia débil (menos del 55\% de consenso general y Kappas inferiores a 0.32); el resto de los 5 ítems presentan una concordancia pobre (menos del $43 \%$ de consenso general y Kappas inferiores a 0.15 ).

Las sanciones para faltas consideradas muy graves con un nivel suficiente de concordancia entre expertos (Kappa de Fleiss > 0,40) fueron tres: a) "p5_c_2. Suspender acto/prueba de evaluación" (K=081); b) "p5_c_3. Suspender la asignatura en este año académico" (K=0,69); y c) "p5_c_7. Pérdida de derecho del alumno/a infractor a ostentar ningún cargo de representación en la universidad" $(\mathrm{K}=0,46)$.

Tabla 5

Sanciones aplicables a faltas consideradas muy graves

\begin{tabular}{lcccccc}
\hline & Desacuerdo & Neutralidad & Consenso & Total & $\begin{array}{c}\text { \% Consenso } \\
\text { General }\end{array}$ & $\begin{array}{c}\text { Free-marginal } \\
\text { Kappa }\end{array}$ \\
\hline $\begin{array}{l}\text { Suspender acto/prueba de } \\
\text { evaluación ante faltas muy } \\
\text { graves }\end{array}$ & 3 & 1 & 58 & 62 & $87,57 \%$ & 0,81 \\
\hline
\end{tabular}




\begin{tabular}{|c|c|c|c|c|c|c|}
\hline & Desacuerdo & Neutralidad & Consenso & Total & $\begin{array}{c}\% \text { Consenso } \\
\text { General }\end{array}$ & $\begin{array}{c}\text { Free-marginal } \\
\text { Kappa }\end{array}$ \\
\hline \multirow{2}{*}{$\begin{array}{l}\text { Suspender la asignatura en este } \\
\text { año académico ante faltas muy } \\
\text { graves }\end{array}$} & 4 & 3 & 55 & 62 & \multirow[t]{2}{*}{$79,01 \%$} & \multirow[t]{2}{*}{0,69} \\
\hline & $6,45 \%$ & $4,84 \%$ & $88,71 \%$ & $100 \%$ & & \\
\hline \multirow{2}{*}{$\begin{array}{l}\text { Pérdida de derecho del } \\
\text { alumno/a infractor a ostentar } \\
\text { ningún cargo de represent- } \\
\text { ación en la universidad ante } \\
\text { faltas muy graves }\end{array}$} & 8 & 5 & 49 & 62 & \multirow[t]{2}{*}{$64,20 \%$} & \multirow[t]{2}{*}{0,46} \\
\hline & $12,90 \%$ & $8,06 \%$ & $79,03 \%$ & $100 \%$ & & \\
\hline \multirow{2}{*}{$\begin{array}{l}\text { Pérdida de derechos de gratui- } \\
\text { dad de créditos ante faltas muy } \\
\text { graves }\end{array}$} & 11 & 7 & 44 & 62 & \multirow[t]{2}{*}{$54,05 \%$} & \multirow[t]{2}{*}{0,31} \\
\hline & $17,74 \%$ & $11,29 \%$ & $70,97 \%$ & $100 \%$ & & \\
\hline \multirow{2}{*}{$\begin{array}{l}\text { Pérdida renovación de becas o } \\
\text { ayudas propias de la universi- } \\
\text { dad ante faltas muy graves }\end{array}$} & 12 & 7 & 43 & 62 & \multirow[t]{2}{*}{$52,35 \%$} & \multirow[t]{2}{*}{0,29} \\
\hline & $19,35 \%$ & $11,29 \%$ & $69,35 \%$ & $100 \%$ & & \\
\hline \multirow{2}{*}{$\begin{array}{l}\text { Prohibición temporal de acceso } \\
\text { a la universidad ante faltas } \\
\text { muy graves }\end{array}$} & 17 & 9 & 36 & 62 & \multirow[t]{2}{*}{$42,41 \%$} & \multirow[t]{2}{*}{0,14} \\
\hline & $27,42 \%$ & $14,52 \%$ & $58,06 \%$ & $100 \%$ & & \\
\hline \multirow{2}{*}{$\begin{array}{l}\text { Amonestación verbal ante } \\
\text { faltas muy graves }\end{array}$} & 29 & 5 & 28 & 62 & \multirow[t]{2}{*}{$41,99 \%$} & \multirow[t]{2}{*}{0,13} \\
\hline & $46,77 \%$ & $8,06 \%$ & $45,16 \%$ & $100 \%$ & & \\
\hline \multirow{2}{*}{$\begin{array}{l}\text { Expulsión de la universidad } \\
\text { ante faltas muy graves }\end{array}$} & 23 & 7 & 32 & 62 & \multirow[t]{2}{*}{$40,72 \%$} & \multirow[t]{2}{*}{0,11} \\
\hline & $37,10 \%$ & $11,29 \%$ & $51,61 \%$ & $100 \%$ & & \\
\hline \multirow{2}{*}{$\begin{array}{l}\text { Revisar evaluaciones anteriores } \\
\text { ante faltas muy graves }\end{array}$} & 27 & 8 & 27 & 62 & \multirow[t]{2}{*}{$38,60 \%$} & \multirow[t]{2}{*}{0,08} \\
\hline & $43,55 \%$ & $12,90 \%$ & $43,55 \%$ & $100 \%$ & & \\
\hline \multirow{2}{*}{$\begin{array}{l}\text { Suspender todas las asigna- } \\
\text { turas matriculadas en este año } \\
\text { académico ante faltas muy } \\
\text { graves }\end{array}$} & 20 & 11 & 21 & 60 & \multirow[t]{2}{*}{$37,55 \%$} & \multirow[t]{2}{*}{0,06} \\
\hline & $3226 \%$ & $1774^{\circ}$ & $5000 \%$ & $100 \%$ & & \\
\hline TOTAL ÍTEMS & & & & & $53,84 \%$ & 0,31 \\
\hline
\end{tabular}

$95 \%$ Intervalo de confianza $[0,14,0,47]$

\section{Discusión y conclusiones}

Las principales conclusiones de nuestro estudio son:

a) De forma global, los resultados obtenidos señalan que la fuerza de la concordancia entre los jueces en las cuestiones analizadas resultó pobre (Kappas globales inferiores a 0,37$)$, lo que muestra la dificultad de establecer estrategias normativas consensuadas para combatir el fraude en las evaluaciones.

b) De los 41 comportamientos fraudulentos analizados, tan solo 9 se pudieron clasificar como muy graves con una concordancia entre expertos aceptable (Kappa de Fleiss > $0,40)$, mientras que los 32 comportamientos fraudulentos restantes presentaron un nivel de concordancia débil o pobre (Kappa de Fleiss $<0,40$ ). Este hecho pone de manifiesto la dificultad de llegar a acuerdos, incluso entre personas con experiencia en ordenación y gestión académica, sobre cómo dimensionar la gravedad y repercusión de los comportamientos deshonestos en los que puede incurrir el alumnado; sobre todo en los casos menos extremos o percibidos como menos graves. Esta situación puede deberse a una falta de tradición en el abordaje de los comportamientos deshonestos del alumnado universitario en España y 
un déficit de estudios y trabajos sobre la cuestión tal y como ya planteaba Comas (2009) hace una década. El escenario no parece haber mejorado sustancialmente desde entonces.

c) Respecto a las faltas leves, ninguna de las 10 sanciones propuestas presentó un nivel de concordancia entre expertos aceptable. Respecto a las sanciones aplicables a faltas graves, solo una resultó tener un nivel aceptable de concordancia entre expertos: "Suspender acto/prueba de evaluación". Respecto a las sanciones aplicables a faltas muy graves, tres de ellas resultaron tener un nivel aceptable de concordancia entre expertos: a) "Suspender acto/prueba de evaluación"; b) "Suspender la asignatura en este año académico"; y c) "Pérdida de derecho del alumno/a infractor a ostentar ningún cargo de representación en la universidad".

d) Si bien de forma global la fuerza de la concordancia entre jueces es baja, algunos ítems analizados sí que mostraron, individualmente, grados elevados de acuerdo entre los expertos. No obstante, vale la pena señalar que los consensos más elevados se obtuvieron tanto en la clasificación de comportamientos como "muy graves" como en las sanciones aplicables a faltas "muy graves", lo que sugiere que las dificultades para alcanzar consensos y las disparidades en los criterios son mayores a la hora de establecer las diferencias entre conductas fraudulentas leves y graves, así como para discernir si una conducta es grave o muy grave, y en las sanciones aplicables en estas situaciones.

Los resultados del presente trabajo pueden ser útiles a la hora de encarar el fraude y la deshonestidad en las evaluaciones en las aulas universitarias españolas a partir de dispositivos normativos. A este respecto, existen evidencias de que con una reglamentación con previsión de sanciones ante conductas probadas de fraude académico se disminuye la comisión de este tipo de actos por parte del alumnado. Es más, cuanto más estricta es la reglamentación menos comisión de prácticas contra la integridad académica se producen (Erzegovac \& Richardson, 2004; McCabe \& Trevino, 1993; McCabe, Trevino \& Butterfield, 1999; LoSchiavo \& Shatz, 2011; Turner \& Beemsterbooer, 2008). Ahora bien -y de ahí la necesidad de abordar el tema desde una perspectiva holística-, la simple existencia de reglamentos no garantiza una mayor honestidad; para que tengan efecto, estos reglamentos deben ser conocidos, tanto por el profesorado como por el alumnado, y, sobre todo, no ser papel mojado: deben ser aplicados (Comas, 2009; McCabe, Butterfield \& Trevino 2003; Tatum \& Schwartz, 2017; Tornos, 2010; Turner \& Beemsterbooer, 2008). Con el cumplimiento de estas tres condiciones (reglamentos estrictos + conocimiento de los mismos por parte de los actores implicados + aplicación de los mismos), la literatura existente sugiere que se produce una disminución de las prácticas académicas deshonestas (Konheim-Kalkstein, Stellmack, \& Shilkey, 2008; LoSchiavo \& Shatz, 2011). Ahora bien, la aproximación holística al fenómeno, a tenor de los resultados del presente estudio, parece harto compleja en nuestro país. Si ya parece difícil alcanzar acuerdos entre responsables de la gestión académica para establecer parámetros de gravedad a las conductas deshonestas cometidas por el alumnado y a las sanciones a aplicar; qué sucedería si se intentaran alcanzar posiciones de consenso tomando como referentes también las opiniones del profesorado sin responsabilidades de gestión y, sobretodo, el punto de vista del alumnado. Sea como fuere, entendemos que es necesario introducir la cuestión de la integridad académica en la agenda de las universidades españolas con el objetivo de generar masa crítica a partir de la cual diseñar estrategias, planes y medidas de actuación. En nuestra opinión, se debe alentar a los administradores y responsables 
de la gestión académica a que examinen sus prácticas institucionales en busca de factores que puedan contribuir a las malas praxis del alumnado en un esfuerzo por desarrollar normas y estrategias para ayudar a prevenir las malas conductas, que socavan la integridad y los valores básicos de las instituciones académicas.

\section{Referencias}

Altman, D.G. (1991). Practical statistics for medical research. Nueva York: Chapman and Hall.

Belter, R.W. y du Pré, A. (2009). A Strategy to Reduce Plagiarism in an Undergraduate Course. Teaching of Psychology, 36(4), 257-261. doi:10.1080/00986280903173165

Brennan, R. L. y Prediger, D. J. (1981). Coefficient Kappa: Some uses, misuses, and alternatives. Educational and Psychological Measurement, 3(41), 687-699. doi:10.1177/001316448104100307

Cebrian-Robles, V., Raposo-Rivas, M. y Sarmiento-Campos, J.A. (2016). ¿Ética o prácticas deshonestas? El plagio en las titulaciones de Educación. Revista de Educación, 374, 161-182.

Chapman, D.W. y Lindner, S. (2016). Degrees of integrity: the threat of corruption in higher education. Studies in Higher Education, 41(2), 247-268. doi:10.1080/03075079 .2014 .927854

Comas, R. (2009). El ciberplagio y otras formas de deshonestidad académica entre el alumnado universitario [tesis doctoral no publicada] Universidad de las Islas Baleares, España.

Comas, R. y Sureda, J. (2016). Prevalencia y capacidad de reconocimiento del plagio académico entre el alumnado del área de economía. El profesional de la información (EPI), 25(4), 616-622. doi:10.3145/epi.2016.jul.11

Comas, R., Sureda, J. y Salva, F. (2010). Academic plagiarism prevalence among Spanish undergraduate students: an exploratory analysis. Biochemia Medica. 20 (3), 301-306. doi:10.11613/bm.2010.038

Comas, R., Sureda, J., Casero, A. y Morey, M. (2011). La integridad académica entre el alumnado universitario español. Estudios Pedagógicos 37(1) 2007-225. doi:10.4067/ s0718-07052011000100011

Curtis, G.J. y Clare, J. (2017). How Prevalent is Contract Cheating and to What Extent are Students Repeat Offenders? Journal of Academic Ethics, 15(2) 115-124. doi:10.1007/ s10805-017-9278-x

Decreto de 8 de septiembre de 1954 por el que se aprueba el Reglamento de disciplina académica de los Centros oficiales de Enseñanza Superior y de Enseñanza Técnica, dependientes del Ministerio de Educación Nacional. Boletín Oficial del Estado, Madrid 12/10/1954, núm. 285. Recuperado de: https://www.boe.es/buscar/act.php?id=BOEA-1954-17807

Denisova-Schmidt, E. (2017). The Challeges of Academic Integrity in Higher Education: Current Trends and Prospects. Boston: The Boston College Center for International Higher Education.

Ercegovac, Z. y Richardson, J.V (2004). Academic Deshonesty, Plagiarism Includes, in the Digital Age: A literature review. College E Research Libraries, 65(4) 301-318. doi:10.5860/ crl.65.4.301 
Estow, S., Lawrence, E. K. y Adams, K. A. (2011). Practice makes perfect: Improving students' skills in understanding and avoiding plagiarism with a themed methods course. Teaching of psychology, 38(4), 255-258. doi:10.1177/0098628311421323

ETINED (2018). South-East European Project on Policies for Academic Integrity. Strasbourg: Council of Europe Publising. Recuperado de: https://bit.ly/2FBxxwX

Fleiss, J. L. (1971). Measuring nominal scale agreement among many raters. Psychological Bulletin, 76(5), 378-382. doi:10.1037/h0031619

Fleiss, J.L. (1981). Statistical methods for rates and proportions. Hoboken: Wiley.

Foltynek, T. (2013). Plagiarism Policies in Spain. Full Report. IPPHEAE Project. Recuperado de: https://bit.ly/2Rv5ary

Gwet, K.L. (2010). Handbook of interrater reliability. Gaithersburg, MD: Advanced Analytics.

Hallak, J. y Poisson, M. (2007). Corrupt schools, corrupt universities: What can be done? París: International Institute for Educational Planning.

Haswell, S., Jubb, P. y Wearing, B. (1999). Accounting students and cheating: A comparative study for Australia, South Africa and the UK. Teaching Business Ethics, 3, 211-239.

Heyneman, S., Kathryn, P., Anderson, H. y Muraliyeva, N. (2008). The cost of corruption in higher education. Comparative Education Review, 52(1), 1-25. doi:10.1086/524367

Hu, G. y Lei, J. (2015). Chinese University Students' Perceptions of Plagiarism. Ethics and Behavior, 25(3), 233-255. doi:10.1080/10508422.2014.923313

Jakobsson, U. y Westergren, A. (2005). Statistical methods for assessing agreement for ordinal data. Scandinavian Journal of Caring Sciences, 19(4), 427-431. doi:10.1111/ j.1471-6712.2005.00368.x

Konheim-Kalkstein, Y. L., Stellmack, M. A. y Shilkey, M. L. (2008). Comparison of Honor Code and Non-Honor Code Classrooms at a Non-Honor Code University. Journal of College and Character, 9(3). doi:10.2202/1940-1639.1115

Kumar, R. y Tripathi, R.C. (2017). An Analysis of the Impact of Introducing the Plagiarism Detection System in an Institute of Higher Education. Journal of Information $\mathcal{E}$ Knowledge Management, 16(2). doi:10.1142/s0219649217500113

Ledwith, A. y Risquez, A. (2008). Using anti-plagiarism software to promote academic honesty in the context of peer reviewed assignments. Studies in Higher Education, 33(4), 371-384. doi:10.1080/03075070802211562

Ley Orgánica 6/2001, de 21 de diciembre, de Universidades. Boletín Oficial del Estado, Madrid, 24-12-2001 núm. 307. Recuperado de: https://www.boe.es/buscar/doc. php?id=BOE-A-2001-24515

LoSchiavo, F. M. y Shatz, M. A. (2011). The impact of an honor code on cheating in online courses. MERLOT Journal of online Learning and Teaching, 7(2), 179-184. Recuperado de: http://jolt.merlot.org/vol7no2/loschiavo_0611.pdf

Ma, Y., McCabe, D.L. y Liu, R. (2013). Students' Academic Cheating in Chinese Universities: Prevalence, Influencing Factors, and Proposed Action. Journal of Academic Ethics, 11(3), 169-184. doi:10.1007/s10805-013-9186-7

Macdonald, R. y Carroll, J. (2006). Plagiarism - A complex issue requiring a holistic institutional approach. Assessment E Evaluation in Higher Education, 31(2), 233-45. doi:10.1080/02602930500262536 
McCabe, D. L. Trevino, L.K. y Butterfield, L. (1999). Academic Integrity in Honor Code and Non-Honor Code Environments, The Journal of Higher Education, 70(2), 211-234. doi:10.2307/2649128

McCabe, D. y Trevino, L. K. (1993). Academic Dishonesty: Honor Codes and Other Contextual Influences. The Journal of Higher Education, 64(5), 522-538. doi:10.1080/0 0221546.1993.11778446

Mohamedbhai, G. (2016). The Scourge of Fraud and Corruption in Higher Education. International Higher Education, 84, 12-14. doi:10.6017/ihe.2016.84.9111

Osipian, A.L. (2007). Corruption in Higher Education: Conceptual approaches and messurement techniques. Research in Comparative and International Education, 2(4), 313-332. doi:10.2304/rcie.2007.2.4.313

Randolph, J. J. (2008). Online Kappa Calculator [Computer software]. Recuperado de: http://justus.randolph.name/kappa.

Real Decreto 1791/2010, de 30 de diciembre, por el que se aprueba el Estatuto del Estudiante Universitario. Boletín Oficial del Estado. Madrid 31-12-2010, núm. 318 Recuperado de: https://www.boe.es/buscar/act.php?id=BOE-A-2010-20147

Sureda-Negre, J., Reynés-Vives, J. y Comas-Forgas, R. (2016). Reglamentación contra el fraude académico en las universidades española. Revista de la Educación Superior, 45(178), 31-44. doi:10.1016/j.resu.2016.03.002

Sweeney, G., Despota, K. y Lindner, S. (Edit.) (2013). Global corruption report: Education. Transparency International. London: Routledge. Recuperado de: https://www.transparency.org/gcr_education

Tatum, H. y Schwartz, B. M. (2017). Honor codes: Evidence based strategies for improving academic integrity. Theory Into Practice, 56(2), 129-135. doi:10.1080/00405841. 2017.1308175

Teixeira, A. y Rocha, M.F. (2006) Academic Cheating in Austria, Portugal, Romania and Spain: A Comparative Analysis, Research in Comparative and International Education, 1(3). doi:10.2304/rcie.2006.1.3.198

Tornos, J. (2010) Reflexiones sobre el régimen disciplinario de los estudiantes universitarios. En: CEDU XIII Encuentro de Defensores Universitarios y III Asamblea general ordinaria de la CEDU. Barcelona, Publicacions i Edicions de la Universitat de Barcelona. pp. 55-59. Recuperado de: https://bit.ly/2VQNSDL

Turner, S. P. y Beemsterboer, P. L. (2003). Enhancing academic integrity: formulating effective honor codes. Journal of Dental Education, 67(10), 1122-1129.

Warrens, M.J. (2010). Inequalities between multi-rater kappas. Advances in Data Analysis and Classification, 4(4), 271-286. doi:10.1007/s11634-010-0073-4

Youmans, R.J. (2011). Does the adoption of plagiarism-detection software in higher education reduce plagiarism? Studies in Higher Education, 36(7), 749-761. doi:10.1080/03075079.2010.523

Fecha de recepción: 16 de enero de 2019.

Fecha de revisión: 4 de febrero de 2019.

Fecha de aceptación: 15 de mayo de 2019. 
